IdeAs

Idées d'Amériques

8 | 2016

Ressources minières dans les Amériques: Mutations

d'un continent

\title{
Ressources minières dans les Amériques: Mutations d'un continent
}

Susanne Berthier-Foglar, Franck Gaudichaud et Sandrine Tolazzi

\section{CpenEdition}

Journals

Édition électronique

URL : https://journals.openedition.org/ideas/1636

DOI : $10.4000 /$ ideas. 1636

ISSN : 1950-5701

Traduction(s) :

Mineral resources in the Americas - a continent under mutation - URL : https:// journals.openedition.org/ideas/2268 [en]

Recursos mineros en las Américas : mutaciones de un continente - URL : https:// journals.openedition.org/ideas/3096 [es]

Recursos minerais nas Américas. Mutações de um continente - URL : https://

journals.openedition.org/ideas/4709 [pt]

Éditeur

Institut des Amériques

Référence électronique

Susanne Berthier-Foglar, Franck Gaudichaud et Sandrine Tolazzi, « Ressources minières dans les Amériques: Mutations d'un continent », IdeAs [En ligne], 8| 2016, mis en ligne le 06 février 2018 consulté le 18 octobre 2022. URL : http://journals.openedition.org/ideas/1636 ; DOI : https://doi.org/ 10.4000/ideas. 1636

Ce document a été généré automatiquement le 18 octobre 2022

\section{c)}

Creative Commons - Attribution - Pas d'Utilisation Commerciale - Pas de Modification 4.0 International - CC BY-NC-ND 4.0

https://creativecommons.org/licenses/by-nc-nd/4.0/ 


\title{
Ressources minières dans les Amériques: Mutations d'un continent
}

\author{
Susanne Berthier-Foglar, Franck Gaudichaud et Sandrine Tolazzi
}

Dans un contexte où l'émergence du réchauffement climatique comme menace globale peine à freiner la surconsommation et la course à l'énergie qui en sont largement la cause, la question du développement des ressources minières et énergétiques se pose avec d'autant plus d'acuité que leur production a fortement augmenté sur le continent américain, comme partout ailleurs dans le monde. Ces dernières années, le «boom » de l'extractivisme, en tant qu'activité humaine capable de modifier de manière significative l'environnement et le climat, peut être considéré en cela comme partie intégrante d'un ensemble de facteurs marquant une nouvelle ère géologique amorcée au tournant du XIX ${ }^{e}$ siècle et baptisée « anthropocène » par le prix Nobel Paul Crutzen. En attribuant une origine humaine aux transformations du climat ou de l'environnement, les tenants de l'anthropocène, tels que Christophe Bonneuil et JeanBaptiste Fressoz dans leur ouvrage L'Événément anthropocène (Paris : Seuil, 2013) invitent à une prise de conscience de l'impact de ces activités sur des changements globaux qui laissent à penser que l'on a désormais atteint un point de non retour. Bien que certains membres de la communauté scientifique refusent encore d'attribuer une origine anthropique à ces changements (Samuele Furfari, dans Sandrine Tolazzi, «Ressources minières et énergétiques: le choc des discours »), une grande majorité d'entre eux insistent sur la nécessité de lier de manière plus générale la question des conséquences environnementales à celle de l'exploitation des ressources (Normand Mousseau, dans le même article). Il est vrai, par ailleurs, que la question environnementale se trouve souvent au cœur des débats actuels sur le développement des ressources minières. Cependant, il semblerait aussi que l'impact économique, politique, géostratégique et social du récent essor des industries extractives sur le continent américain entraîne de véritables mutations à l'échelle de ce continent, comme le souligne l'entretien avec Bernadette Mérenne-Schoumaker. C'est donc avant tout pour tenter de déterminer la nature de ces mutations que les coordinateurs de ce numéro ont, dans un premier 
temps, organisé un colloque international sur la question des ressources minières dans les Amériques (Grenoble, juin 2014) puis, dans un second temps, fait appel à certains participants à ce colloque, ainsi que d'autres auteurs, afin de constituer un dossier qu'ils espèrent représentatif des questionnements scientifiques actuels. La lecture des différentes contributions, du dossier "Débats", et des entretiens autour de ces questions fait ainsi émerger de nombreuses zones de tension liées à des jeux de pouvoir entre différents acteurs (communautés locales, entreprises, gouvernements à différentes échelles, etc.) mais également à des représentations de ces jeux de pouvoir parfois liées à l'histoire du développement minier en général.

2 La problématique de l'exploitation minière - et plus largement des ressources naturelles - est au cœur des grands bouleversements géopolitiques et de la redéfinition asymétrique des rapports nord-sud : l'appropriation, l'extraction et le contrôle des richesses que cette activité signifie a été l'un des puissants moteurs des processus coloniaux et d'expansion territoriale qu'ont connu les Amériques depuis les XV-XVI ${ }^{\mathrm{e}}$ siècles. La « soif » de l'or et de l'argent ont alimenté une expérience de longue durée de domination éco-sociale, politique et culturelle, par-delà même les Indépendances, forgeant jusqu'à nos jours une "tradition" de type colonial d'exploitation des ressources par le biais duquel les économies latino-américaines extraverties dépendent des grandes puissances industrielles qui dominent le système international (voir «Domination et extraversion de l'Amérique latine » dans Géopolitique des Amériques, dir. Alain Musset, Paris : Nathan, 2014, $4^{\mathrm{e}}$ ed.). Ce modèle se retrouve entre le nord et le sud du continent, mais aussi dans les relations de l'Amérique latine avec d'autres pôles de l'économie mondiale (Europe, Chine), encore au plan interne au sein même des pays, au sud comme du nord du continent : ainsi Lucie Genay («L'extraction d'uranium au Nouveau-Mexique: un symbole de la conquête par l'atome») montre à quel point l'histoire de l'extraction de l'uranium au Nouveau-Mexique confirme que, si les enjeux miniers ont changé, certains schémas néocoloniaux de la construction nationale étasunienne sont restés les mêmes et perpétuent un héritage d'inégalité sociale et raciale, générant par là même des réactions contrastées des nations indiennes concernées. Ce «nécolonialisme extractiviste » est également dénoncé avec force par nombre de mouvements sociaux-environnementaux et communautés indigènes en Amérique latine, mobilisations qui sont à l'origine de plusieurs conflits tant avec les gouvernements en place qu'avec les entreprises minières. Face à la prolifération de ces mobilisations, les exécutifs de l'ensemble du continent s'essaient d'ailleurs à plusieurs stratégies, allant de la répression ou de la contention institutionnelle jusqu'aux moratoires et à la déclaration d'une partie du territoire national comme libre d'exploitation minière (Maria Teresa Gutierrez-Haces, "L'investissement étranger et l'activité minière en Amérique latine. Les stratégies gouvernementales dans les conflits avec les compagnies minières »). Car, qu'ils se disent progressistes, "nationauxpopulaires » ou conservateurs, l'ensemble des gouvernements ont essayé de mettre à profit le «boom " du prix des matières premières et minières des dernières années, bien que selon des logiques souvent différentes en termes de politiques publiques. Néanmoins, la crise mondiale et la chute de la demande depuis 2007-2008 ont souligné jusqu'à quel point les économies américaines pouvaient s'inscrire dans une logique de rente et de reprimarisation, contraire à toute perspective de développement « soutenable " ou vertueux. Un autre modèle minier est-il alors possible? C'est ce que suggère le gouvernement bolivarien du Venezuela avec son « Plan d'Action Stratégique commun pour le Développement de la Ceinture de l'Orénoque et de l'Arc minier » qui 
prétend articuler croissance économique, alliance avec les entreprises multinationales et développement des services sociaux publics, tout en "contrôlant » l'évolution du front pionnier minier. Néanmoins, l'étude de Anne Péné-Annette sur l'extraction aurifère dans l'Etat Bolívar ( La relance de l'extraction minière dans la Guyane vénézuélienne?») rappelle l'ampleur des «destructions environnementales et des dégâts humains » que cet extractivisme mené ici à l'échelle de tout un pays peut signifier.

3 Désormais nombre de chercheurs et activistes proposent non seulement des analyses critiques sur ces formes d'exploitation industrielle de la nature, mais aussi mettent en avant des modèles alternatifs de relation entre la Nature, la société et l'économie ou encore entre le nord et le sud (Sandrine Tolazzi, « Ressources minières et énergétiques: le choc des discours ", et l'article de Maristella Svampa ou l'ouvrage d'Anna Bednik cités dans Franck Gaudichaud, "Ressources minières, "extractivisme " et développement en Amérique latine: perspectives critiques »). Comme en Équateur, le concept du Bien-vivre (le Sumak Kawsay quechua) tout comme l'idée de laisser particulièrement dans des zones de forte biodiversité - les minerais et le pétrole sous la terre commencent à gagner du terrain dans la société civile et dans le champ politique, sans pour autant que soit résolue la tension problématique entre protection des écosystèmes, respects des communautés et accès à un certain niveau de vie pour toutes et tous. Ainsi, dans la Bolivie d'Evo Morales, si l'imaginaire du «Buen Vivir » reste une référence symbolique toujours mobilisée par le Président, le pays reste fortement dépendant de l'exploitation des ressources minières et gazières, dont le développement actuel répond parfois davantage aux pressions de différentes organisations corporatistes qu'à la reconquête de la souveraineté nationale annoncée (Claude Le Gouill, "Néo-développementalisme, «buen vivir» et luttes corporatistes dans la politique minière du gouvernement d'Evo Morales, Bolivie »).

4 La question environnementale fait également partie intégrante d'une histoire du développement minier qui, selon Michel Deshaies, a toujours revêtu un caractère " prédateur » pour l'environnement (« Mines et environnement dans les Amériques: les paradoxes de l'exploitation minière »). Certes, les petits prospecteurs et entreprises minières exploitant sans retenue les ressources dans l'optique d'une rentabilité immédiate et laissant ensuite à l'abandon des terrains hautement pollués ont disparu au profit de grandes sociétés minières dont l'activité est désormais mieux encadrée. Par ailleurs, les pressions de certaines communautés locales et d'ONG ont contraint ces entreprises à réduire leur impact environnemental tout en s'impliquant davantage dans le développement des territoires concernés afin d'augmenter leur " acceptabilité » et tenter de renverser l'image négative qu'elles peuvent projeter. Deshaies note ainsi que, paradoxalement, les grandes entreprises minières rencontrent une opposition croissante en raison de leur impact environnemental, alors que même que ce dernier n'a jamais été aussi réduit. Il n'en reste pas moins que l'échelle même des projets miniers actuellement en développement fait que cet impact reste important, sans compter qu'il faut également prendre en compte les problèmes liés à la réhabilitation des territoires une fois leur exploitation terminée ou encore le risque de catastrophes accidentelles (ainsi que vient de le rappeler récemment, et de manière dramatique, la catastrophe minière de Mariana, dans l'État brésilien du Minas Gerais).

5 Le débat autour des conséquences environnementales des méga-projets miniers fait donc rage, poussant les gouvernements à prendre davantage de précautions par le biais 
d'études telles que l'évaluation environnementale stratégique sur les gaz de schistes commandée par le gouvernement du Québec, et à laquelle Christiane Gagnon et Majella-J Gauthier ont participé («Inventaire et vulnérabilité des basses-terres du Saint-Laurent (Québec, Canada) face à l'exploitation du gaz de schiste »). Le travail que leur étude présente a ainsi permis d'identifier des zones de vulnérabilité, ce qui devrait favoriser une meilleure gestion du territoire dans le cadre de projets d'exploitation au Québec. Cependant, il reste difficile pour les scientifiques de se positionner en-dehors de tout discours par rapport à de tels enjeux. En effet, on peut distinguer très nettement une rhétorique visant à minimiser l'impact des grands projets de développement, voire à les présenter comme bénéfiques pour l'environnement. Ainsi, non seulement Samuele Furfari affirme-t-il que «l'énergie fossile, en dépit de son impact environnemental, a eu des répercussions extraordinairement positives sur nos conditions de travail, de vie et de santé », mais il présente également le développement du gaz de schiste comme une solution pour réduire les gaz à effets de serre (Sandrine Tolazzi, «Ressources minières et énergétiques: le choc des discours »). Dès lors, les opposants au développement du gaz de schiste, par exemple, sont présentés comme des personnes mal informées sur les processus d'exploration et d'extraction (RTI International, dans le même article). A l'inverse, on peut distinguer un discours qui fait très clairement état de l'impact environnemental du développement des ressources minières et le présente comme un des principaux défis à relever dans l'élaboration d'une stratégie mondiale des ressources minières (Normand Mousseau, dans le même article). La question est alors de savoir s'il faut se concentrer sur la réduction de l'impact environnemental des projets miniers ou sur la suppression pure et simple de ces projets dans le cadre d'une transition écologique.

6 Par ailleurs, l'exploitation des ressources mobilise de plus en plus d'acteurs locaux qui prennent conscience de leur situation de minorité exploitée, voire sacrificielle. La localisation de gisements dans des zones de montagne, qui étaient historiquement le refuge de populations pauvres, le plus souvent autochtones, a fait prendre conscience à ces habitants de régions minières qu'ils étaient spoliés et qu'ils l'avaient été de longue date. Les ethnicités locales prennent alors une nouvelle visibilité dans le champ politique dès lors qu'elles s'opposent aux décideurs d'un État perçu comme faisant partie d'un autre groupe social et culturel. En Amérique Latine, on observe la résurgence des anciennes identités ethniques, mises en veille dans un contexte d'assimilation et de dénigrement des «Indiens », un terme qui, en espagnol (Indio), s'apparente souvent à une injure. Depuis une quinzaine d'années, les communautés paysannes, qui sont parfois des autochtones, au sens où l'entend la Déclaration des Droits des Peuples Autochtones de l'ONU, opposent une résistance croissante aux projets miniers et exigent des entreprises minières des retombées locales plus importantes ainsi qu'une participation aux bénéfices. L'article de Carmen Salazar-Soler («La place de l'ethnicité dans les conflits miniers socio-environnementaux dans les Andes du Pérou, $\mathrm{XX}^{\mathrm{e}}-\mathrm{XXI}^{\mathrm{e}}$ siècles ») met en évidence cette prise de conscience et les demandes des peuples autochtones en matière de contrôle effectif de la pollution et de participation aux choix concernant l'exploitation et les usages des ressources naturelles de leurs territoires.

7 Pour Lucie Genay (« L'extraction d'uranium au Nouveau-Mexique, un symbole de la conquête par l'atome »), cette industrie extractive sur les terres des autochtones et des Hispaniques du Nouveau-Mexique s'apparente à la fois à une conquête et à une colonisation. Elle met en évidence la vulnérabilité des habitants des régions minières 
dont l'économie est fragilisée par le caractère cyclique de l'exploitation de ces ressources. D'un point de vue social, elle relève aussi la persistance d'un héritage de dépendance et d'inégalités malgré le militantisme fort, mais sans cohésion centralisatrice, qui s'organise dans les régions où sont localisés les gisements d'uranium.

8 Le militantisme écologique est ancré de longue date dans l'Ouest américain où il prend son origine dans les mouvements de conservation de la nature et de création des premiers parcs nationaux à la fin du XIXème siècle. Cette tradition d'une vision de la nature se retrouve dans le militantisme d'opposition aux exploitations minières. Un lieu à forte visibilité environnementale, qu'il s'agisse d'un parc national ou autre zone protégée, voire d'une montagne à caractère sacré, cristallise les oppositions à l'exploitation minière. C'est particulièrement évident dans les opinions très marquées des tribus/nations Navajo et Laguna (Susanne Berthier-Foglar, "L'exploitation des ressources minières de l'Ouest américain », et Lucie Genay, "L'extraction d'uranium au Nouveau-Mexique »).

9 Dépassant le cadre étasunien, les organisations militantes à visée internationale s'intéressent aux situations de spoliation des peuples opprimés dans le monde. Loin du contexte social et économique, ces prises de position n'ont pas toujours un grand effet mais ont le mérite d'alerter l'opinion publique. De toute façon, dans les pays concernés, il est bien évident que les raisons économiques ou militaires priment sur la protection de la nature. Dans Susanne Berthier-Foglar ( « Ressources minières et énergétiques: Évolution et constance des défis »), on voit comment il a été possible pour les propriétaires d'une mine d'uranium, au cœur du parc national du Grand Canyon, de faire accepter une exploitation intrusive sur le bord de la falaise, à quelques centaines de mètres d'un point de vue touristique. Pendant la Guerre froide, les enjeux de la défense nationale avec la course à l'armement atomique semblaient plus importants que l'intégrité d'un parc.

10 Ainsi, la question de l'exploitation des ressources minières et énergétiques est centrale dans la compréhension de certains modèles économiques de développement mais aussi des rapports de force qui sont en jeu. Elle est d'autant plus fascinante pour les chercheurs qui s'y intéressent qu'elle soulève des questions à toutes les échelles, de l'individu - comme on peut l'observer à travers les photographies de Louie Palu (entretien avec Lucie Genay) - à la communauté, en passant par les acteurs gouvernementaux et industriels, avec un impact économique et environnemental pouvant se mesurer à l'échelle locale mais aussi régionale, voire globale. Il s'agit donc d'une question véritablement stratégique au sein des Amériques, les articles sélectionnés ici constituant une première présentation de certains aspects liés à ce vaste champ de recherche. 


\section{AUTEURS}

\section{SUSANNE BERTHIER-FOGLAR}

Susanne Berthier-Foglar est professeur, spécialiste de l'Ouest américain et des peuples autochtones, Université Grenoble Alpes (ILCEA4). susanne.berthier@univ-grenoble-alpes.fr

\section{FRANCK GAUDICHAUD}

Franck Gaudichaud est maître de conférences en histoire et civilisation de l'Amérique Latine à l'Université Grenoble Alpes et membre de l'ILCEA4 (EA 7356). franck.gaudichaud@univ-grenoblealpes.fr

\section{SANDRINE TOLAZZI}

Sandrine Tolazzi est maître de conférences à l'Université Grenoble Alpes (UFR des Langues, ILCEA4 - Institut des Langues et des Cultures d'Europe, Amérique, Afrique, Asie et Australie). sandrine.tolazzi@univ-grenoble-alpes.fr 